\title{
Estudo preliminar da síntese de geopolímeros baseados em metacaulim, cinzas volantes e filito da Região Sudeste do Pará
}

Este trabalho apresenta um estudo preliminar da síntese de geopolímeros com matérias primas e coprodutos provenientes da região Sul e Sudeste do Pará, no qual foram definidas 4 formulações para avaliação da resistência mecânica através de ensaios de resistência à compressão. Os insumos inicialmente escolhidos foram: metacaulim, filito, cinza volante e escória de aciaria; estes dois últimos sendo coprodutos industriais. Na confecção das formulações preliminares foi utilizado como ativador alcalino a solução de $\mathrm{NaOH} 12 \mathrm{M}$, bem como o silicato de sódio (Na2SiO3) como solução aditivadora. Entre as amostras ensaiadas, duas

formulações obtiveram resistência à compressão da ordem de 20,5 e $21 \mathrm{MPa}$, com respectivamente 15 e 28 dias de cura, com adição do silicato de sódio.

Palavras-chave: Geopolímero; Ativação alcalina; Metacaulim; Cinzas volantes.

\section{Preliminary study of the synthesis of metakaolin-based geopolymers, fly ash and phyllyte of the Southeast Region of Pará}

This study presents a preliminary evaluation of the synthesis of geopolymers with raw materials and co-products from South and Southeast of Pará State - Brazil, in which 4 formulations for mechanical strength evaluation through compressive strength tests were defined. The inputs initially chosen were: metakaolin, phyllite, steel slag and fly ash; the latter two being industrial co-products. In the preparation of the preliminary formulations, $\mathrm{NaOH} 12 \mathrm{M}$ solution was used as alkaline activator, as well as sodium silicate ( $\mathrm{Na2SiO3)}$ as additive solution. Among the tested samples, two formulations obtained compressive strength of 20.5 and 21 $\mathrm{MPa}$, with respectively 15 and 28 curing days, with the addition of sodium silicate.

Keywords: Geopolymer; Alkaline activation; Metakaolin; Fly ash.

Topic: Engenharia Civil

Reviewed anonymously in the process of blind peer.
Received: 05/11/2019

Approved: $10 / 12 / 2019$
Gilciene Guevara Duarte Cardoso (iD

Universidade Federal do Sul e Sudeste do Pará, Brasil http://lattes.cnpq.br/3959090222850284

http://orcid.org/0000-0002-3909-4337

cardososgv@gmail.com

Renata Lilian Ribeiro Portugal Fagury

Universidade Federal do Sul e Sudeste do Pará, Brasi

http://lattes.cnpq.br/4758312798406543

http://orcid.org/0000-0002-1144-1106

renatafagury@unifesspa.edu.br

Adriano Alves Rabelo (iD)

Universidade Federal do Sul e Sudeste do Pará, Brasi

http://lattes.cnpq.br/6564133385744551

http://orcid.org/0000-0003-3953-3149

adriano@unifesspa.edu.br
Elias Fagury Neto (iD)

Universidade Federal do Sul e Sudeste do Pará, Brasil

http://lattes.cnpq.br/3004502388905443

http://orcid.org/0000-0003-2346-509X

fagury@unifesspa.edu.br
Referencing this:

CARDOSO, G. G. D.; FAGURY, R. L. R. P.; RABELO, A. A.; FAGURY NETO, E.. Estudo preliminar da síntese de geopolímeros baseados em metacaulim, cinzas volantes e filito da Região Sudeste do Pará. Technology Science, v.2, n.1, p.37-42, 2020. DOI:

http://doi.org/10.6008/CBPC2674-6425.2020.001.0007 


\section{INTRODUÇÃO}

O geopolímero é uma classe de ligante polimérico inorgânico tipicamente sintetizado via ativação alcalina de precursores de aluminossilicato (DAVIDOVITS, 1989). Este material tem atraído interesse crescente nas últimas décadas, devido ao seu potencial promissor para ser usado como um ligante alternativo ao cimento Portland comum. Embora o cimento Portland tenha sido amplamente utilizado durante séculos com desempenhos superiores de engenharia, sua produção contribui com $5 \%$ a $8 \%$ das emissões de $\mathrm{CO}_{2}$ em todo o mundo (SCRIVENER et al., 2008). Os geopolímeros, por outro lado, são feitos principalmente a partir de subprodutos industriais ou outros materiais aluminossilicatos baratos e a produção de geopolímeros geralmente impacta menos o meio ambiente (LI et al., 2019).

Ao contrário do geopolímero, o cimento Portland que é comumente utilizado, não possui um caráter sustentável. Sendo ele responsável por grandes emissões de $\mathrm{CO}_{2}$, gás que se acredita ser um grande intensificador do efeito estufa (ANTUNES, 2013). Segundo Torgal et al. (2005), desde 2005 iniciou-se uma nova fase de valorização ambiental, onde iniciou-se uma rejeição a materiais com alto teor de emissão de gases nocivos, como é o caso do cimento Portland, onde a cada tonelada de cimento fabricada, produz-se uma tonelada de $\mathrm{CO}_{2}$, assim superando a emissão de gases nocivos de ligantes geopolímericos em 7 vezes.

Segundo Davidovits (1994), geopolímeros são inorgânicos, resistentes, estáveis até $1250^{\circ} \mathrm{C}$ e nãoinflamáveis, o que o nomeou a chave para o desenvolvimento de novos materiais, resultando no impulso à criatividade e inovação. Para Rocha et al. (2018), geopolímeros são adequados principalmente para construção civil, devido ao seu potencial para substituir derivados do cimento Portland, resultado de suas excelentes propriedades físico-mecânicas, durabilidade e aderência ao ferro e concreto comum.

A respeito do processo de geopolimerização, é necessária uma fonte de aluminossilicatos e uma solução alcalina, Palomo et al. (1999) afirmam que a ativação alcalina é o que permite a transformação de estruturas amorfas e/ou metaestáveis em compósitos compactos e com características cimentantes. Segundo Severo et al. (2013) há um mecanismo geral de ativação alcalina em materiais constituídos por sílica e alumina. O mecanismo consiste em 4 etapas: dissolução, coagulação, condensação e cristalização. A primeira etapa ocorre com a quebra das ligações covalentes $\mathrm{Si}-\mathrm{O}-\mathrm{Si}$ e $\mathrm{Al}-\mathrm{O}-\mathrm{Si}$, devido ao $\mathrm{pH}$ elevado da solução alcalina; após a quebra das ligações há formação de uma dispersão coloidal. Posteriormente, há o acúmulo de produtos da quebra das ligações que interagem entre si, formando uma estrutura coagulada. A terceira fase conduz a geração de uma estrutura condensada e, por fim, com influência do tempo e temperatura, inicia-se a etapa de cristalização. O processo de geopolimerização é demonstrado na Figura 1.

Em relação a acréscimo de $\mathrm{Na}_{2} \mathrm{SiO}_{3}$ (silicato de sódio), o mesmo influência de forma positiva o processo de polimerização, pois a sílica reage com a alumina livre na solução, e dessa forma origina um produto com maior presença de Si e mais resistente. Como Pinto (2004) relata, a ativação alcalina do metacaulim com $\mathrm{Na}_{2} \mathrm{SiO}_{3}$ pode resultar em um aumento de resistência mecânica na compressão e flexão em, respectivamente 30 a $60 \mathrm{MPa}$ e 5 a $7 \mathrm{MPa}$. 


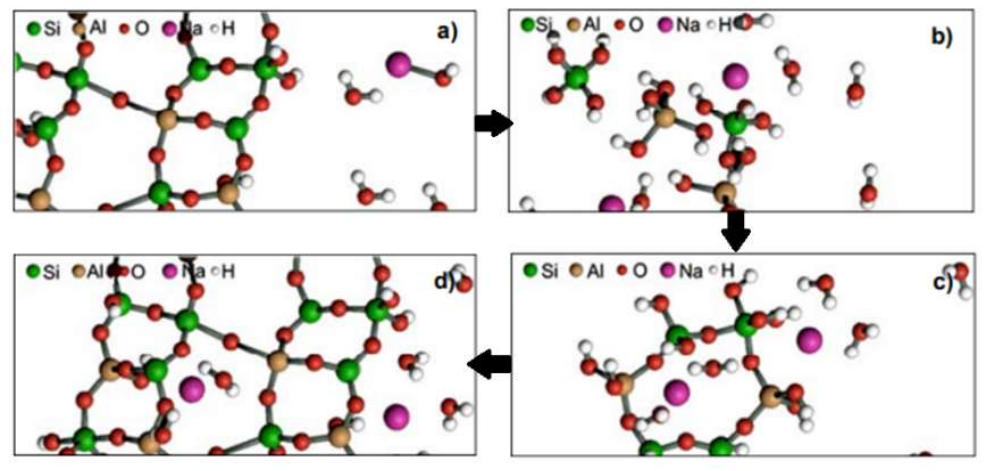

Figura 1: Mecanismo geral de ativação alcalina em aluminossilicatos.

Fonte: Adaptado de Severo et al. (2013).

\section{METODOLOGIA}

Inicialmente, foram definidas as matérias primas a serem utilizadas no projeto. O metacaulim foi produzido a partir da calcinação do caulim processado (Ymeris, Brasil). O filito utilizado foi proveniente da região de Marabá/PA; a cinza volante, material fonte mais comum de sílica, foi proveniente de uma olaria local, que foi resultante da queima de madeira para geração de calor. A escória de forno panela foi obtida de uma siderúrgica local. É importante ressaltar que o filito e o caulim passaram por tratamentos térmicos a 700 oC durante 4 horas. A calcinação do caulim resultou em metacaulim, o qual apresenta propriedades pozolânicas.

Metacaulim, filito e escória passaram por um processamento prévio de moagem, para alcançar a granulometria adequada para peneiramento em uma peneira de 200 mesh Tyler $(74 \mu \mathrm{m})$. A solução ativadora utilizada foi de $\mathrm{NaOH} 12 \mathrm{M}$ (C.R.Q). Para o preparo de cada formulação da 1a etapa foram utilizadas apenas as matérias primas e a solução ativadora ( $90 \mathrm{ml}$ para $140 \mathrm{~g}$ de matéria-prima) e na 2 a etapa foram utilizados os mesmos constituintes da etapa 1 , com adição da solução de $\mathrm{Na}_{2} \mathrm{SiO}_{3}$; os componentes de ambas as etapas foram misturados manualmente, até apresentarem o aspecto homogêneo. Foram definidas 4 formulações que foram utilizadas para a primeira etapa e dessas quatro formulações apenas duas foram utilizadas na segunda etapa, para realizar a síntese de geopolímeros, como demonstra a Tabela 1:

Tabela 1: Formulações para a síntese de geopolímeros.

\begin{tabular}{|l|l|l|l|l|}
\hline & Filito (\%) & Escória (\%) & MK (\%) & FA (\%) \\
\hline I & X & X & 50 & 50 \\
\hline II & 25 & X & 37,5 & 37,5 \\
\hline III & X & 25 & 37,5 & 37,5 \\
\hline IV & 12,5 & 12,5 & 37,5 & 37,5 \\
\hline
\end{tabular}

Para a segunda etapa foram repetidas as formulações descritas na Tabela 1 com o acréscimo de $\mathrm{Na}_{2} \mathrm{SiO}_{3}$ (silicato de sódio) inicialmente numa proporção de 30\% da quantidade de $\mathrm{NaOH}$ necessário para 140 g de matéria-prima, ou seja $27 \mathrm{ml}$. Em seguida, as misturas foram colocadas moldes de PVC, de $4 \mathrm{~cm}$ de altura e $2 \mathrm{~cm}$ de diâmetro, conforme demonstram as Figuras 2 e 3, para adquirir uma conformação adequada para o ensaio de compressão. Este procedimento está de acordo com a norma NBR 5739. 


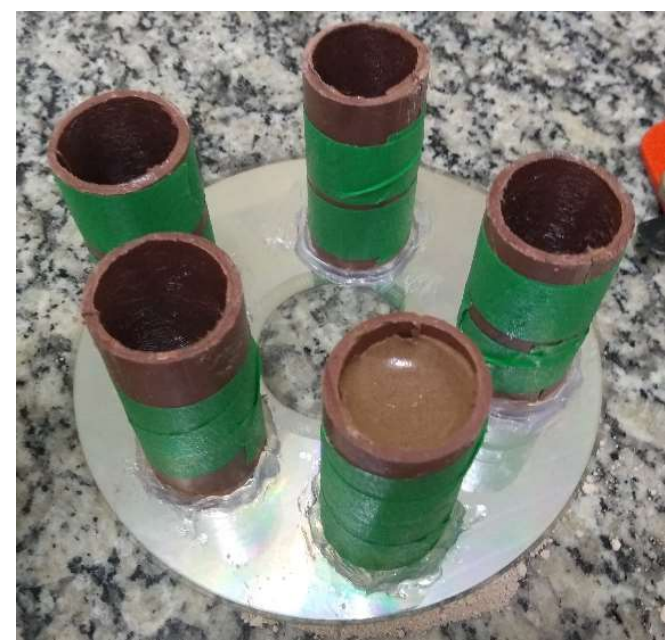

Figura 2: Conformação dos corpos de prova.

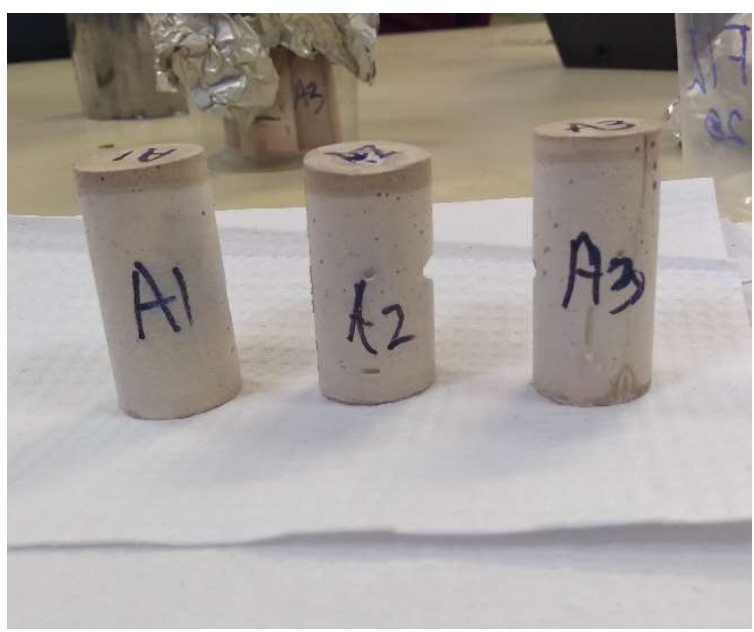

Figura 3: Corpos de prova conformados da formulação (Etapa 2) no tempo de cura de 28 dias.

\section{RESULTADOS E DISCUSSÃO}

Durante o preparo do geopolímero percebeu-se bastante resistência à dissolução da matéria-prima em relação a adição de solução de $\mathrm{NaOH}$ : à medida que foi sendo adicionado mais solução ativadora, a matéria-prima dissolveu-se com maior facilidade até apresentar um aspecto condensado, como mostra a Figura 4; já em misturas com $\mathrm{N}_{2} \mathrm{SiO}_{3}$, o aspecto apresentado foi mais coeso e homogêneo ao contrário de misturas sem $\mathrm{N}_{2} \mathrm{SiO}_{3}$, como mostra a Figura 5 .

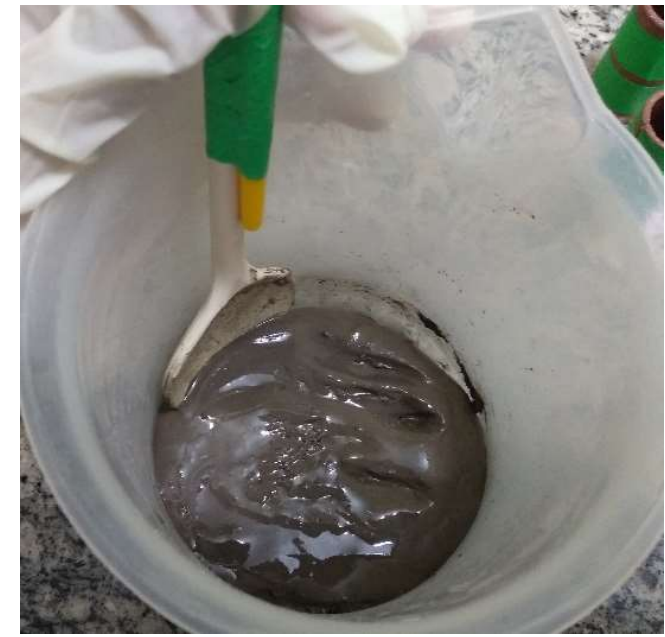

Figura 4: Mistura homogênea para formação do geopolímero (Formulação III).

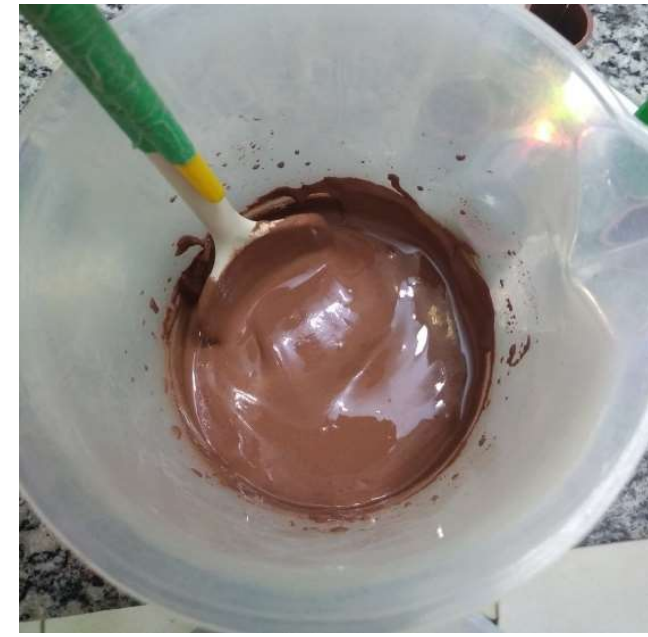

Figura 5: Mistura homogênea 2aetapa (FII).

Inicialmente, o objetivo foi avaliar a adequação das matérias-primas à confecção de geopolímeros se utilizando uma solução ativadora alcalina. Os resultados da resistência à compressão dos corpos de prova de geopolímeros da 1a etapa mostraram que as formulações sem adição de escória de aciaria (I e II) apresentaram melhores desempenhos, apesar das falhas na conformação dos corpos de prova, que apresentaram fissuras e bolhas de ar, assim afetando seu desempenho. Este fato pode ser devido à melhor dissolução das estruturas dos aluminossilicatos presentes no metacaulim, cinza e filito, e uma maior dificuldade de dissolução dos componentes presentes na escória, que é um coproduto de composição muito complexa, podendo conter por exemplo óxidos de cálcio, ferro, zinco, entre outros (FREITAS, 2018). 
Tabela 2: Resultados de resistência à compressão das formulações de geopolímeros, em função da idade de cura (Etapa 1).

\begin{tabular}{|l|l|l|l|l|l|}
\hline \multicolumn{5}{|l|}{ Formulação I (MPa) } & \multicolumn{4}{l|}{ Formulação II (MPa) } \\
\hline 7 dias & 15 dias & 28 dias & 7 dias & 15 dias & 28 dias \\
\hline $\mathbf{5 , 5 1}$ & $\mathbf{1 0 , 4 3}$ & $\mathbf{1 1 , 8 2}$ & $\mathbf{4 , 3 4}$ & $\mathbf{9 , 3 4}$ & $\mathbf{1 3 , 1 2}$ \\
\hline Formulação III (MPa) & $\mathbf{3 4}$ & Formulação IV (MPa) & $\mathbf{2 8}$ dias \\
\hline $\mathbf{7}$ dias & $\mathbf{1 5}$ dias & $\mathbf{2 8}$ dias & $\mathbf{7}$ dias & $\mathbf{1 5}$ dias & $\mathbf{7 , 3 1}$ \\
\hline $\mathbf{9 , 3 8}$ & $\mathbf{9 , 0 3}$ & $\mathbf{1 0 , 9 1}$ & $\mathbf{5 , 8 5}$ & $\mathbf{4 , 5 4}$ & \\
\hline
\end{tabular}

Na segunda etapa foram utilizadas as formulações I e II com acréscimo de $30 \%$ de $\mathrm{Na}_{2} \mathrm{SiO}_{3}$, sendo obtido respectivamente os seguintes resultados dos ensaios de compressão.

Tabela 3: Resultados de resistência à compressão das formulações de geopolímeros, em função da idade de cura (Etapa 2).

\begin{tabular}{|l|l|l|l|l|l|}
\hline Formulação I (MPa) & \multicolumn{4}{l|}{ Formulação II (MPa) } \\
\hline 7 dias & 15 dias & 28 dias & 7 dias & 15 dias & 28 dias \\
\hline 7,33 & 10,33 & 13,33 & $\mathbf{1 1 , 3 3}$ & $\mathbf{2 0 , 5}$ & $\mathbf{2 1}$ \\
\hline
\end{tabular}

Percebeu-se, com a adição do silicato de sódio, que se obteve um aumento de resistência à compressão de acordo com o aumento do tempo de cura e comparando os resultados da formulação I e II da 1 a e $2^{\text {a }}$ etapa (com a adição de $\mathrm{Na}_{2} \mathrm{SiO}_{3}$ ) obteve-se um aumento na resistência a compressão. Nas Figuras 6 e 7, estão representados o ensaio de compressão e o aspecto do corpo de prova depois de ensaiado.

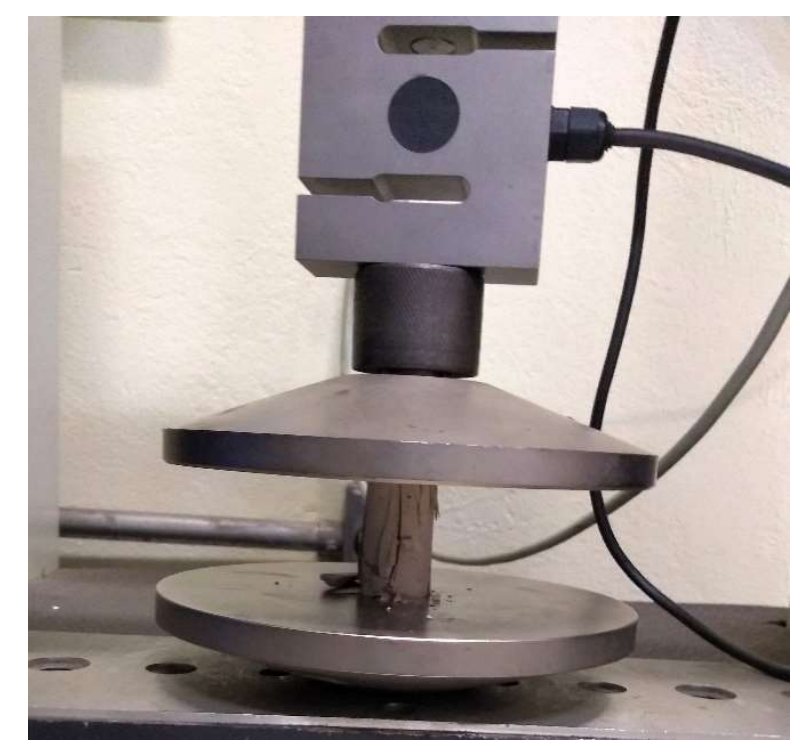

Figura 6: Ensaio da formulação I (Etapa 1) ensaio (tempo de cura: 7 dias).

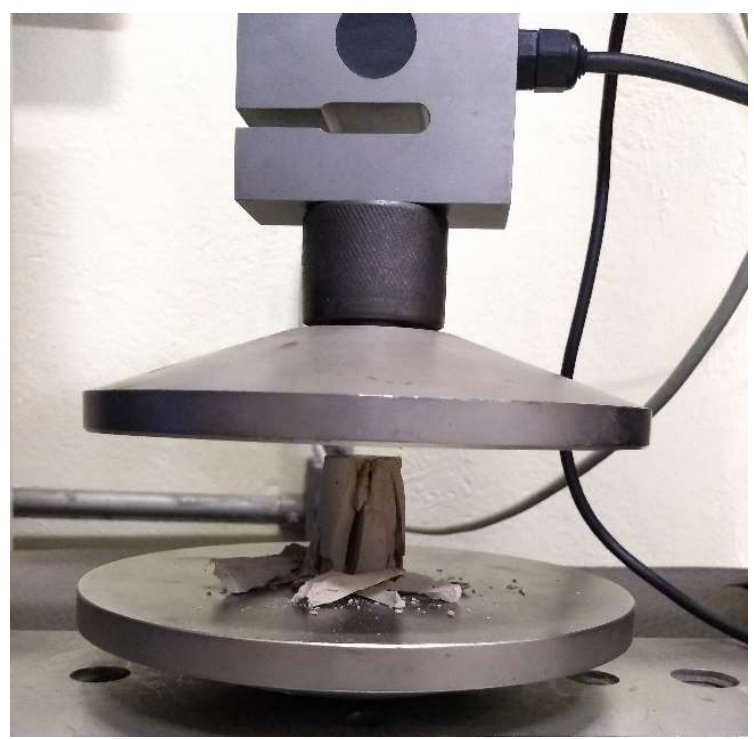

Figura 7: Aspecto da formulação I (Etapa 1) após (tempo de cura: 7 dias).

Os resultados indicam que estes materiais chegaram a uma resistência mecânica próxima ao cimento Portland que, segundo Bauer (2000), possui uma resistência mínima de 25 MPa aos 28 dias. Logo, os materiais obtidos nesta pesquisa podem ser empregados como substitutos de materiais à base de cimento Portland, em aplicações que não requeiram elevada solicitação mecânica, como por exemplo em reparos de fachadas azulejadas, fissuras em paredes de alvenaria, etc..

\section{CONCLUSÕES}

Com os resultados obtidos foi possível averiguar que o geopolímero possui capacidade de substituir 
o cimento Portland em funções não estruturais, devido ao sucesso de sintetização a partir de matérias-primas oriundas do Sudeste do Pará, apresentando potencial para desenvolvimento de formulações maior resistência mecânica. Dentre as formulações da etapa 1, as que continham escória de aciaria demonstraram uma menor resistência mecânica em relação às demais. A segunda etapa, que possui como característica a adição de solução aditivadora de $\mathrm{Na}_{2} \mathrm{SiO}_{3}$, foi iniciada com as formulações I e II, que em seus primeiros ensaios de compressão já apresentaram uma melhoria na resistência mecânica em relação às mesmas formulações da etapa anterior, assim apresentado resultados da ordem de 20,5 e $21 \mathrm{MPa}$, nas respectivas idades de 15 e 28 dias de cura, da formulação II. No entanto, é importante ressaltar que os resultados obtidos podem ter sido afetados por falha na consolidação das amostras, pois as mesmas apresentavam bolhas de ar e fissuras.

\section{REFERÊNCIAS}

ANTUNES, E.. Obtenção de Geopolímero a partir de metacaulim ativado. Dissertação (Mestrado em Construção Civil) - Universidade Federal de Minas gerais, Belo Horizonte, 2013.

BAUER, L. A. F.. Materiais de construção. 5 ed. Rio de Janeiro: LTC, 2000.

DAVIDOVITS, J.. Geopolymer and geopolymeric materials. J. Therm. Anal., v.35, p.429-441, 1989.

DAVIDOVITS, J.. Geopolymers: Man- made rock geosynthesis and the resulting development of very early high strength cement. Journal of Materials Education, v.16, n.2/3, p.91139, 1994

FREITAS, S. M. A. C.. Escória de Aciaria: Caminhos para uma Gestão Sustentável. Tese (Doutorado em Engenharia de Materiais) - Universidade Federal de Ouro Preto, Ouro Preto, 2018.

LI, Z.; ZHANG, S.; ZUO, Y.; CHEN, W.; YE, G.. Chemical deformation of metakaolin based geopolymer. Cement and Concrete Research, v.20, p.108-118, 2019. DOI: http://doi.org/10.1016/i.cemconres.2019.03.017

PALOMO, A. M. W.; GRUTZECK, M. W.; BLANCO, M. T.. Alkali-activated fly ashes: A cement for future. Cem. Concr. Res., v.29, n.8, p.1323-1329, 1999.

\section{DOI: http://doi.org/10.1016/S0008-8846(98)00243-9}

PINTO, A. T.. Sistemas ligantes obtidos por activação alcalina de metacaulino. Tese (Doutorado em Engenharia Civil) - Universidade do Minho, Braga, 2004

ROCHA, T. S.; DIAS, D. P.; FRANÇA, F. C. C.; GUERRA, R. R. S.; MARQUES, L. R. C. O.. Metakaolin-based geopolymer mortars with different alkaline activators ( $\mathrm{Na}+$ and $\mathrm{K}+$ ). Construction and Building Materials, v.178, p.453-461, 2018. DOI:

http://doi.org/10.1016/j.conbuildmat.2018.05.172

SCRIVENER, K. L.; KIRKPATRICK, R. J.. Innovation in use and research on cementitious material. Cem. Concr. Res., v.38, p.128-136, 2008

DOI: http://doi.org.10.1016/i.cemconres.2007.09.025

SEVERO, C. G. S.; COSTA, D. L.; BEZERRA, I. M. T.; MENEZES, R. R.; NEVES, G. A.. Características, particularidades e princípios científicos dos materiais ativados alcalinamente. Revista Eletrônica de Materiais e Processos, v.8, n.2, p.5567, 2013.

TORGAL, F. P.; CASTRO-GOMES, J. P.; JALALI, S.. Cimento Portland Normal Versus Ligantes Geopoliméricos: Considerações Econômicas Sobre as Implicações do Mercado do Carbono no Custo dos Betões. Lisboa: Instituto Politécnico de Castelo Branco, 2005.

A CBPC - Companhia Brasileira de Produção Científica (CNPJ: 11.221.422/0001-03) detém os direitos materiais desta publicação. Os direitos referem-se à publicação do trabalho em qualquer parte do mundo, incluindo os direitos às renovações, expansões e disseminações da contribuiç̃o, bem como outros direitos subsidiários. Todos os trabalhos publicados eletronicamente poderão posteriormente ser publicados em coletâneas impressas sob coordenação da Sapientiae Publishing, da Companhia Brasileira de Produção Científica e seus parceiros autorizados. Os (as) autores (as) preservam os direitos autorais, mas não têm permissão para a publicação da contribuição em outro meio, impresso ou digital, em português ou em tradução. 